\title{
On Topological Singular Set of Maps with Finite 3-Energy into $S^{3}$
}

\author{
M. R. Pakzad
}

\begin{abstract}
We prove that the topological singular set of a map in $W^{1,3}\left(M, \mathbb{S}^{3}\right)$ is the boundary of an integer-multiplicity rectifiable current in $M$, where $M$ is a closed smooth manifold of dimension greater than 3 and $\mathbb{S}^{3}$ is the three-dimensional sphere. Also, we prove that the mass of the minimal integer-multiplicity rectifiable current taking this set as the boundary is a strongly continuous functional on $W^{1,3}\left(M, \mathbb{S}^{3}\right)$.

Keywords: Topological singularities, minimal connections, flat chains and rectifiable currents, Sobolev spaces between manifolds
\end{abstract}

AMS subject classification: 28A75, 46E35, 46E40, 49Q15

\section{Introduction}

Let $M$ be an oriented smooth closed Riemannian manifold of dimension $n$, and $N$ any closed Riemannian manifold isometricly embedded into $\mathbb{R}^{N}$. Let

$$
W^{1, p}(M, N)=\left\{u \in W^{1, p}\left(M, \mathbb{R}^{N}\right): u(x) \in N \text { a.e. on } M\right\} .
$$

For $u \in W^{1, p}(M, N)$ the $p$-energy is given by the functional

$$
E(u)=\int_{M}|\nabla u|^{p} d \operatorname{vol}_{M} .
$$

In [6], F. Bethuel and X. Zheng proved that smooth maps are not strongly dense in $W^{1, p}(M, N)$ if $p<n$ and $\pi_{[p]}(N) \neq 0,[p]$ being the integer part of $p$. In this case, one may want to characterize the maps in $W^{1, p}(M, N)$ which are approximable by smooth maps and identify the obstruction for maps which are not. Precisely, we would like to associate to any map $u \in W^{1, p}(M, N)$ a topological singular set $\mathbf{S}_{u}$ which characterizes the approximability of $u$ by smooth maps, i.e. $u$ would be the strong limit of smooth maps if and only if $\mathbf{S}_{u}=0$.

In this line, F. Bethuel proved in [3] that $u \in W^{1,2}\left(\mathbb{B}^{n}, \mathbb{S}^{2}\right)$ is strongly approximable by maps in $C^{\infty}\left(\mathbb{B}^{n}, \mathbb{S}^{2}\right)$ if and only if $d\left(u^{*} \omega_{S^{2}}\right)=0$ in the sense of distributions, where $\mathbb{B}^{n}$ is the $n$-dimensional unit disk and $\mathbb{S}^{2}$ is the two-dimensional sphere. The same result holds for the space $W^{1, p}\left(\mathbb{B}^{n}, \mathbb{S}^{p}\right)$ for any other integer $p$ (see [5]). Thus, the "local" topological obstruction for maps in $W^{1, p}\left(M, \mathbb{S}^{[p]}\right)$ can be defined as a current:

Mohammad Reza Pakzad: Ecole Normale Supérieure de Cachan, Centre de Math. \& de Leurs Appl., CNRS, URA 1611, 61 Av. du Président Wilson, F-94235 Cachan Cedex

Present address: Max-Planck-Inst. for Math. in the Sci., Inselstr. 22 - 26, D-04103 Leipzig pakzad@cmla.ens-cachan.fr and pakzad@mis.mpg.de 
Definition 1. Let $p<n$ and $u \in W^{1, p}\left(M, \mathbb{S}^{[p]}\right)$. The topological singular set of $u$, $\mathbf{S}_{u} \in \mathcal{D}_{n-[p]-1}(M)$, is the current defined by

$$
\mathbf{S}_{u}(\alpha)=\int_{M} u^{*} \omega \wedge d \alpha \quad\left(\alpha \in \mathcal{D}^{n-[p]-1}(M)\right) .
$$

Here $\mathcal{D}^{k}(M)$ is the set of smooth $k$-forms on $M$ with compact support (see [14: Vol. $\mathrm{I} /$ Section 2.2.3]) and $\omega$ is any $[p]$-form on $\mathbb{S}^{[p]}$ for which $\int_{\mathbb{S}[p]} \omega=1$.

Remark 1. Recent developments by F. Hang and F. H. Lin [15] showed that the condition $\mathbf{S}_{u}=0$, though being necessary for the strong approximability of a map $u \in W^{1, p}\left(M, \mathbb{S}^{p}\right)$ by smooth maps in this space, is not always sufficient due to some obstructions lying in the "global" topological structure of certain domains. Precisely, there is a map $u \in H^{1}\left(\mathbb{C P}^{2}, \mathbb{S}^{2}\right)$ for which $d\left(u^{*} \omega\right)=0$ while $u$ is not in the strong closure of smooth maps in $H^{1}\left(\mathbb{C P}^{2}, \mathbb{S}^{2}\right)$.

Two important problems about $\mathbf{S}_{u}$ for $u \in W^{1, p}\left(M, \mathbb{S}^{p}\right)$ are still open for almost every integer $p$. First, we do not know whether $\mathbf{S}_{u}$ is always the boundary of an integermultiplicity rectifiable current, i.e. if it is an integral flat chain. This has been proved for $p=1$ and $p=n-1$ (see [14: Vol. II/Section 5.4.3]) and $p=2$ (see [19]). The second problem arises if the answer to the first one is positive. Set for $\mathbf{S}$, any integral flat chain in $M$ of dimension $k$,

$$
m_{i}(\mathbf{S})=\inf \left\{\mathbf{M}(\mathbf{T}): T \in \mathcal{R}_{k+1}(M) \text { and } \partial \mathbf{T}=\mathbf{S}_{u}\right\},
$$

the minimal mass of integer-multiplicity rectifiable currents taking $\mathbf{S}$ as the boundary. Then the question would be to determine whether $m_{i}\left(\mathbf{S}_{u_{m}}-\mathbf{S}_{u}\right) \rightarrow 0$ if $u_{m} \rightarrow u$ strongly in $W^{1, p}\left(M, \mathbb{S}^{p}\right)$. The answer is yes for $p=1$ and $p=n-1$ (see [4] and [14: Vol. II/Section 5.4.2]), while we do not know whether this is the case for the maps in $H^{1}\left(\mathbb{B}^{4}, \mathbb{S}^{2}\right)$. We encounter this case when considering the problem of relaxing the Dirichlet energy for maps into $\mathbb{S}^{2}$. As we saw in [19], generalizing to higher dimensions the algebraic formula given in [4] for the relaxed Dirichlet energy from a 3-dimensional domain into $\mathbb{S}^{2}$ is possible if we prove that $m_{i}\left(\mathbf{S}_{u}\right)$ is strongly continuous on $H^{1}\left(\mathbb{B}, \mathbb{S}^{2}\right)$.

Another case where the second problem shows its importance is when we try to define a topological singular set for maps in $W^{1, p}\left(\mathbb{B}^{n}, N\right)$. In [5], F. Bethuel, J. M. Coron, F. Demengel and F. Helein gave a description of this set for when $N$ is $([p]-1)$ connected and $\pi_{[p]}(N)$ is torsion-free. Considering the problem for when $\pi_{[p]}(N)$ has torsion, the author and T. Rivière remarked that we can define this set as a flat $\pi_{[p]}(N)$ chain if these two questions come to have a positive answer for $[p]$. As an example, the topological singular set of any map in $u \in W^{1,1}\left(\mathbb{B}^{n}, \mathbb{R P}\right)$ is a flat $\mathbb{Z}_{2}$-chain, and is equal to zero if and only if $u$ is the strong limit of smooth maps in $W^{1,1}\left(\mathbb{B}^{n}, \mathbb{R P}^{2}\right.$ ) (see [20]).

In this paper we solve these problems for $p=3$ and $p=7$. The particularity of these two cases reside in the fact that $\mathbb{S}^{3}$ and $\mathbb{S}^{7}$ (alongside with $\mathbb{S}^{1}$ ) are the only spheres which have this property: There is a smooth multiplication

$$
\kappa: \mathbb{S}^{k} \times \mathbb{S}^{k} \rightarrow \mathbb{S}^{k}
$$

such that the induced homotopic homeomorphism

$$
\kappa_{*}: \pi_{k}\left(\mathbb{S}^{k}\right) \oplus \pi_{k}\left(\mathbb{S}^{k}\right) \rightarrow \pi_{k}\left(\mathbb{S}^{k}\right)
$$


is the sum of elements in $\pi_{k}\left(\mathbb{S}^{k}\right)$. As a result, the method we use does not work for other values of $p$.

Here is our main result:

Theorem 1. Let $p=3$ or $p=7, p<n=\operatorname{dim} M$ and $u \in W^{1, p}\left(M, \mathbb{S}^{p}\right)$. Then $\mathbf{S}_{u}$ is the boundary of an integer-multiplicity rectifiable current in M. Moreover, $m_{i}\left(\mathbf{S}_{u_{m}}-\right.$ $\left.\mathbf{S}_{u}\right) \rightarrow 0$ if $u_{m} \rightarrow u$ strongly in $W^{1, p}\left(M, \mathbb{S}^{p}\right)$.

If $M$ is not closed, we set

$$
W_{\varphi}^{1, p}(M, N)=\left\{u \in W^{1, p}(M, N): u=\varphi \text { on } \partial M\right\}
$$

where $\varphi$ is a given boundary data. We assume that $\varphi$ is in $C^{\infty}(\partial M, N)$ and can be extended into $M$ by a smooth map. Then we have

Theorem 1 bis. Let $p=3$ or $p=7, p<n=\operatorname{dim} M$ and $u \in W_{\varphi}^{1, p}\left(M, \mathbb{S}^{p}\right)$. Then $\mathbf{S}_{u}$ is the boundary of an integer-multiplicity rectifiable current in $M$. Moreover, $m_{i}\left(\mathbf{S}_{u_{m}}-\mathbf{S}_{u}\right) \rightarrow 0$ if $u_{m} \rightarrow u$ strongly in $W_{\varphi}^{1, p}\left(M, \mathbb{S}^{p}\right)$.

Considering the question of topological singular sets, using the methods of [20] we have the following corollaries. The readers can be referred to $[13,20,21]$ for definitions and more details.

Corollary 1. Let $\mathbb{B}^{n}$ be the $n$-dimensional unit disk, $n>[p]=3$ or $n>[p]=7$, and assume that $N$ is a closed $([p]-1)$-connected Riemannian manifold of dimension equal or greater than $[p]$. Then $\mathbf{S}_{u}$, the topological singular set of any $u \in W^{1, p}\left(\mathbb{B}^{n}, N\right)$, is well defined as a flat $\pi_{[p]}(N)$-chain and the flat norm of $\mathbf{S}_{u_{m}}-\mathbf{S}_{u}$ converges to 0 if $u_{m} \rightarrow u$ in $W^{1, p}\left(\mathbb{B}^{n}, N\right)$. Moreover, $u$ is the strong limit of smooth maps in $W^{1, p}\left(\mathbb{B}^{n}, N\right)$ if and only if $\mathbf{S}_{u}=0$.

Remark 2. The cases where $N$ is not $([p]-1)$-connected are more involved. The readers can be referred to [16], where $\mathrm{T}$. Rivière and $\mathrm{R}$. Hardt have treated the relatively difficult case of $W^{1,3}\left(\mathbb{B}^{4}, \mathbb{S}^{2}\right)$.

Corollary 1 bis. Let $\mathbb{B}$ be the $n$-dimensional unit disk, $n>[p]=3$ or $n>[p]=7$, and assume that $N$ is a closed $([p]-1)$-connected Riemannian manifold of dimension equal or greater than $[p]$. We assume also that $\varphi \in C^{\infty}(\partial \mathbb{B}, N)$ is smoothly extendable into $\mathbb{B}$. Then $u$ is the strong limit of smooth maps in $W_{\varphi}^{1, p}(\mathbb{B}, N)$ if and only if $\mathbf{S}_{u}=0$.

\section{Some known facts}

In this section we present some classic definitions and theorems regarding the singularities of maps in Sobolev spaces between manifolds.

Definition 2. We say that $u \in W^{1, p}\left(M, \mathbb{S}^{p}\right)$ is in $R^{\infty, p}\left(M, \mathbb{S}^{p}\right)$ if $u$ is smooth except on $B=\cup_{i=1}^{m} \sigma_{i} \cup B_{0}$, a compact subset of $M$, where $\mathcal{H}^{n-p-1}\left(B_{0}\right)=0$ and $\sigma_{i}$ are smooth embeddings of the unit disk of dimension $n-p-1$. Moreover, we assume that any two different faces of $B, \sigma_{i}$ and $\sigma_{j}$, may meet only on their boundaries.

Theorem 2 (Bethuel [2]). $R^{\infty, p}\left(M, \mathbb{S}^{p}\right)$ is dense in $W^{1, p}\left(M, \mathbb{S}^{p}\right)$ for the strong topology.

We recall the definition of the topological singular set $\mathbf{S}_{u}$ of $u$ : 
Definition 3. Let $u \in W^{1, p}\left(M, \mathbb{S}^{p}\right)$. We define the current $\mathbf{S}_{u} \in \mathcal{D}_{n-p-1}(M)$ to be the current defined by

$$
\mathbf{S}_{u}(\alpha)=\int_{M} u^{*} \omega \wedge d \alpha \quad\left(\alpha \in \mathcal{D}^{n-p-1}(M)\right)
$$

where $\mathcal{D}^{k}(M)$ is the set of smooth $k$-forms on $M$ with compact support (see [14: Vol. $\mathrm{I} /$ Section 2.2.3]) and $\omega$ is some $p$-form on $\mathbb{S}^{p}$ for which $\int_{\mathbb{S}^{p}} \omega=1$.

Let $\omega_{1}$ and $\omega_{2}$ be two such forms on $\mathbb{S}^{p}$. We have $\omega_{1}-\omega_{2}=d \beta$ where $\beta$ is some smooth 1-form on $\mathbb{S}^{p}$ extendable to $\mathbb{R}^{p+1}$. Let $u \in W^{1, p}\left(M, \mathbb{S}^{p}\right)$ and consider a sequence $u_{m} \in C^{\infty}\left(M, \mathbb{R}^{p+1}\right)$ converging to $u$ in $W^{1, p}$. We have

$$
u_{m}^{*}(d \beta)=d\left(u_{m}^{*} \beta\right),
$$

and by passing to the limit, we observe that this holds true for $u$ in the sense of distributions. This proves the independence of $\mathbf{S}_{u}$ from the choice of $\omega$ as we have

$$
d\left(u^{*} \omega_{1}\right)-d\left(u^{*} \omega_{2}\right)=d u^{*}(d \beta)=0
$$

in the sense of distributions. Now the existence of the integral (1) is a direct consequence of the inequality

$$
\left|u^{*} \omega\right| \leq \frac{1}{p^{\frac{p}{2}} \alpha_{p}}|\nabla u|^{p} \quad \text { a.e. on } M
$$

where $\alpha_{p}=\left|\mathbb{S}^{p}\right|$ and $\alpha_{p} \omega=\omega_{V}$ is the standard volume form of $\mathbb{S}^{p}$.

We shall give a description of $\mathbf{S}_{u}$ for $u \in R^{\infty, p}\left(M, \mathbb{S}^{p}\right)$. Clearly, if $u$ is smooth, a standard operation on pull-back yields $d\left(u^{*} \omega\right)=u^{*}(d \omega)=0$ and as a consequence we deduce for $u \in R^{\infty, p}\left(M, \mathbb{S}^{p}\right)$ that $\mathbf{S}_{u} \subseteq B$.

Definition 4. Let $u \in R^{\infty, p}\left(M, \mathbb{S}^{p}\right)$ and let $B=\cup \sigma_{i} \cup B_{0}$ be the singular set of $u$. Suppose that each $\sigma_{i}$ is oriented by a smooth $(n-p-1)$-vector field $\vec{\sigma}_{i}$. For $a \in \sigma_{i}$ let $N_{a}$ be any $(p+1)$-dimensional smooth submanifold of $M$, orthogonal to $\sigma_{i}$ at $a$. Consider the embedded $(p+1)$-disk $M_{a, \delta}=B_{\delta}(a) \cap N_{a}$ oriented by the $(p+1)$-vector field $\vec{M}_{a}$ such that $(-1)^{n-p} \vec{\sigma}_{i}(a) \wedge \vec{M}_{a}$ is the fixed orientation of $M$. Then the topological degree of $u$ on the $p$-dimensional topological sphere $\Sigma_{a, \delta}=\partial M_{a, \delta}$ is well defined and is independent of the choice of $a$ and $N_{a}$ for $\delta$ small enough. We call this integer the degree of $u$ on $\sigma_{i}$ and denote it by

$$
\operatorname{deg}_{\sigma_{i}} u \text {. }
$$

Remember that any $k$-dimensional rectifiable subset $\mathcal{M}$ of $M$ considered with a multiplicity $\theta$ and oriented by a unit $k$-vector field $\xi$ defines a rectifiable current as

$$
\tau(\mathcal{M}, \theta, \xi)(\alpha)=\int_{\mathcal{M}}\langle\xi, \alpha\rangle \theta d \mathcal{H}^{k} \quad\left(\alpha \in \mathcal{D}^{k}(M)\right) .
$$

We should recall some useful results. 
Lemma 1. If $\left\{u_{m}\right\}$ is a sequence of maps in $W^{1, p}\left(M, \mathbb{S}^{p}\right)$ converging to $u$, then $\mathbf{S}_{u_{m}} \rightarrow \mathbf{S}_{u}$ in the sense of currents, i.e. for any smooth $(n-p-1)$-form $\alpha$ in $M$ we have

$$
\mathbf{S}_{u}=\lim _{m \rightarrow \infty} \mathbf{S}_{u_{m}}(\alpha)
$$

Equivalently,

$$
m_{r}\left(\mathbf{S}_{u_{m}}-\mathbf{S}_{u}\right) \rightarrow 0 \quad \text { if } u_{m} \rightarrow u \text { in } W^{1, p}\left(M, \mathbb{S}^{p}\right)
$$

where $m_{r}(\mathbf{S})$ is the minimal mass of normal currents taking $\mathbf{S}$ as their boundary.

Lemma 2. Let $M$ be a compact Riemannian manifold. Then for any $u \in R^{\infty, p}\left(M, \mathbb{S}^{p}\right)$, $\mathbf{S}_{u}$ is the integer-multiplicity rectifiable current

$$
\sum_{i=1}^{m}\left(\operatorname{deg}_{\sigma_{i}} u\right) \tau\left(\sigma_{i}, 1, \vec{\sigma}_{i}\right)
$$

Meanwhile, if $\partial M$ is empty, or if $\left.u\right|_{\partial M}$ is homotopic to a constant, then $\mathbf{S}_{u}$ is the boundary of some integer-multiplicity rectifiable current of finite mass.

The reader can find the proofs of these statements for the case $p=2$ in [18, 19], $M$ being a domain in $\mathbb{R}^{n}$. The proofs are essentially the same for other values of $p$ and any smooth compact manifold.

Remark 3. By Lemma 1, Theorem 1 would come true for any $p$ if $\frac{m_{i}(\mathbf{S})}{m_{r}(\mathbf{S})}<C$ for any integral flat $(n-p-1)$-chain $\mathbf{S}$ in $M$. The existence of such a constant $C$ is an open problem except for when $\operatorname{dim} \mathbf{S}=0$ or $\operatorname{dim} \mathbf{S}=n-2$, where we have the equality $m_{i}(\mathbf{S})=m_{r}(\mathbf{S})$ for any integral flat chain. Refer to [1], [8: Lemma 4.2], [10: Section 3 and Appendix], [12: Theorem 5.10] and [14: Vol. II/Section 1.3.4] for proofs and different aspects of the problem.

Theorem 3 (Almgren, Browder and Lieb [1]). Let $M$ be as above and let $u \in$ $R^{\infty, p}\left(M, \mathbb{S}^{p}\right)$ such that either $\partial M$ is empty or $\left.u\right|_{\partial M}$ is constant. Then

$$
m_{i}\left(\mathbf{S}_{u}\right) \leq \frac{1}{p^{\frac{p}{2}} \alpha_{p}} \int_{M}|\nabla u|^{p} d \operatorname{vol}_{M}
$$

\section{Proof of Theorem 1}

We identify $\mathbb{S}^{3}$ (respectively $\mathbb{S}^{7}$ ) with the unit spheres in quaternions (respectively Cayley numbers) and observe that they inherit the product structure on these spaces. If we show the quaternion product (respectively, the Cayley product) by $\kappa(x, y)=x \circ y$, $\kappa$ will be a smooth map from $\mathbb{S}^{k} \times \mathbb{S}^{k} \rightarrow \mathbb{S}^{k}$ for $k=3$ or $k=7$ and will satisfy the condition that the induced homotopic homeomorphism

$$
\kappa_{*}: \pi_{k}\left(\mathbb{S}^{k}\right) \oplus \pi_{k}\left(\mathbb{S}^{k}\right) \rightarrow \pi_{k}\left(\mathbb{S}^{k}\right)
$$

is the sum of elements in $\pi_{k}\left(\mathbb{S}^{k}\right)$. The spheres of dimensions $0,1,3$ and 7 are the only spheres for which such $\kappa$ exist (see [7: Section VI.15, p. 412]). By $x^{-1} \in \mathbb{S}^{k}$ we mean the right inverse of $x \in \mathbb{S}^{k}$. Set

$$
u \circ v^{-1}(x)=u(x) \circ v(x)^{-1}
$$

for $u, v \in W^{1, p}\left(M, \mathbb{S}^{p}\right)$ and $x \in M$. 
Lemma 3. Let $u, v \in W^{1, p}\left(M, \mathbb{S}^{p}\right)$ for $p=3$ or $p=7$. Then $u \circ v^{-1} \in W^{1, p}\left(M, \mathbb{S}^{p}\right)$. Moreover, if $\left\{u_{m}\right\}$ is a strongly convergent sequence in $W^{1, p}\left(M, \mathbb{S}^{p}\right)$, then $E\left(u_{m} \circ u_{k}^{-1}\right) \rightarrow$ 0 if $m, k \rightarrow+\infty$.

Proof. Straight computations show that

$$
\nabla\left(u \circ v^{-1}\right)=\nabla u \circ v^{-1}-u \circ\left(v^{-1} \circ\left(\nabla v \circ v^{-1}\right)\right)
$$

which yields

$$
\left|\nabla\left(u \circ v^{-1}\right)\right| \leq|\nabla u|+|\nabla v|
$$

as $|u|=|v|=1$. Thus $u \circ v^{-1} \in W^{1, p}\left(M, \mathbb{S}^{p}\right)$. The smoothness of operations and the Lebesgue dominant convergence yields the second part of the lemma

Lemma 4. If $u, v \in R^{\infty, p}\left(M, \mathbb{S}^{p}\right)$ for $p=3$ or $p=7$, then $u \circ v^{-1} \in R^{\infty, p}\left(M, \mathbb{S}^{p}\right)$ and

$$
\mathbf{S}_{u \circ v^{-1}}=\mathbf{S}_{u}-\mathbf{S}_{v}
$$

Proof. That $u \circ v^{-1} \in R^{\infty, p}\left(M, \mathbb{S}^{p}\right)$ is a direct result of smoothness of the product. Relation (3) can be deduced from Lemma 2 and the fact that for any $(n-p-1)$ dimensional face of $B\left(u \circ v^{-1}\right)$ we have $\operatorname{deg}_{\sigma}\left(u \circ v^{-1}\right)=\operatorname{deg}_{\sigma} u-\operatorname{deg}_{\sigma} v$

Now we present the

Proof of Theorem 1. Let $u \in W^{1, p}\left(M, \mathbb{S}^{p}\right)$ for $p=3$ or $p=7$. By Theorem 2 there exists a sequence of maps $u_{m} \in R^{\infty, p}\left(M, \mathbb{S}^{p}\right)$ such that $u_{m} \rightarrow u$ in $W^{1, p}\left(M, \mathbb{S}^{p}\right)$. By Lemma 3 there exist a subsequence $u_{m_{k}}$ of $\left\{u_{m}\right\}$ such that

$$
E\left(u_{m_{k}} \circ u_{m_{k+1}}^{-1}\right) \leq \frac{p^{\frac{p}{2}} \alpha_{p}}{2^{k+1}} .
$$

Meanwhile, using Theorem 3 and (3) we observe that there is an integer-multiplicity rectifiable current $\mathbf{L}_{k}$ such that

$$
\partial \mathbf{L}_{k}=\mathbf{S}_{u_{m_{k}} \circ u_{m_{k+1}}^{-1}}=\mathbf{S}_{u_{m_{k}}}-\mathbf{S}_{u_{m_{k+1}}} \quad \text { and } \quad \mathbf{M}\left(\mathbf{L}_{k}\right) \leq \frac{1}{2^{k}}
$$

Choose a finite mass integer-multiplicity rectifiable current $\mathbf{L}_{0}$ such that $\partial \mathbf{L}_{0}=\mathbf{S}_{u_{m_{1}}}$ and put $\mathbf{L}=\mathbf{L}_{0}-\sum_{i=1}^{+\infty} \mathbf{L}_{i}$. So $\mathbf{M}(\mathbf{L})<+\infty$ and $\mathbf{L}$ is also an integer-multiplicity rectifiable current. Observe that if $\mathbf{I}_{k}:=\mathbf{L}_{0}-\sum_{i=1}^{k} \mathbf{L}_{i}$, then $\partial \mathbf{I}_{k}=\mathbf{S}_{u_{m_{k+1}}}$. Meanwhile $\mathbf{M}\left(\mathbf{I}_{k}-\mathbf{L}\right) \rightarrow 0$. This, using Lemma 1, yields $\partial \mathbf{L}=\mathbf{S}_{u}$ (so far we have proved that $\mathbf{S}_{u}$ is the boundary of some integer-multiplicity rectifiable current in $M$ ). Moreover,

$$
m_{i}\left(\mathbf{S}_{u_{m_{k+1}}}-\mathbf{S}_{u}\right) \leq \mathbf{M}\left(\mathbf{I}_{k}-\mathbf{L}\right) \rightarrow 0 \quad(k \rightarrow+\infty) .
$$

Consequently, for any convergent sequence $u_{m} \in R^{\infty, p}\left(M, \mathbb{S}^{p}\right)$,

$$
m_{i}\left(\mathbf{S}_{u_{m}}-\mathbf{S}_{u}\right) \rightarrow 0 .
$$

As a result, for any $u \in W^{1, p}\left(M, \mathbb{S}^{p}\right), m_{i}\left(\mathbf{S}_{u}\right) \leq C E(u)$ for a constant $C>0$ independent of $u$. Meanwhile, by the strong density of $R^{\infty, p}\left(M, \mathbb{S}^{p}\right)$ in $W^{1, p}\left(M, \mathbb{S}^{p}\right)$ and Lemma 1 , Lemma 4 is true for maps in $W^{1, p}\left(M, \mathbb{S}^{p}\right)$, too. Using the same method and the proved facts about $\mathbf{S}_{u}$, we can prove (4) for any convergent sequence $u_{m} \in W^{1, p}\left(M, S^{p}\right)$, i.e. $m_{i}\left(\mathbf{S}_{u_{m}}-\mathbf{S}_{u}\right) \rightarrow 0$ if $u_{m} \rightarrow u$ in $W^{1, p}\left(M, \mathbb{S}^{p}\right)$ 
Theorem 1 bis can be proved following the same ideas.

Acknowledgement. The author is grateful to Tristan Rivière for having drawn his attention to this problem. This research was carried out with support provided by the French government in the framework of cooperation programs between Université de Versaille and I.P.M., Institute for Studies in Theoretical Physics and Mathematics, Iran.

\section{References}

[1] Almgren, F. J., Browder, W. and E. H. Lieb: Co-area, liquid crystals, and minimal surfaces. Lect. Notes Math. 1306 (1988), 1 - 22.

[2] Bethuel, F.: The approximation problem for Sobolev maps between two manifolds. Acta Math. 167 (1992), 153 - 206.

[3] Bethuel, F.: A characterization of maps in $H^{1}\left(B^{3}, S^{2}\right)$ which can be approximated by smooth maps. Ann. Inst. Henri Poincaré 7 (1990), 269 - 286.

[4] Bethuel, F., Brezis, H. and J.-M. Coron: Relaxed energies for harmonic maps. In: Variational Methods (eds.: H. Berestycki et al.). Basel et al.: Birkhäuser Verlag 1990, pp. 17 -53 .

[5] Bethuel, F., Coron, J.-M., Demengel, F. and F. Hélein: A cohomological criterion for density of smooth maps in Sobolev spaces between two manifolds. In: Nematics, Mathematical and Physical Aspects (NATO ASI Series C 332; eds.: J.-M. Coron et al.). Dordrecht: Kluwer Acad. Publ. 1991, pp. 15 - 23.

[6] Bethuel, F. and X. Zheng: Density of smooth functions between two manifolds in Sobolev spaces. J. Funct. Anal. 80 (1988), $60-75$.

[7] Bredon, G. E.: Topology and Geometry. New York: Springer-Verlag 1993.

[8] Brezis, H., Coron, J.-M. and E. Lieb: Harmonic maps with defects. Comm. Math. Phys. 107 (1986), $649-705$.

[9] Demengel, F. and R. Hadiji: Relaxed energy for functionals on $W^{1,1}\left(B^{2}, S^{1}\right)$. Nonlin. Anal. - Theory, Meth. \& Appl. 19 (1992), 625 - 641.

[10] Evans, L. C.: Partial differential equations and Monge-Kantorovich mass transfer. In: Current developments in mathematics 1997. Papers from the conference held in Cambridge, MA, USA, 1997 (ed.: R. Bott et al.). Boston, MA: Intern. Press 19990, pp. 65 126.

[11] Federer, H.: Geometric Measure Theory. Berlin: Springer-Verlag 1969.

[12] Federer, H.: Real flat chains, cochains and variational problems. Ind. Univ. Math. J. 24 (1974), $351-407$.

[13] Fleming, W.: Flat chains over a coefficient group. Trans. Amer. Math. Soc. 121 (1966), $160-186$.

[14] Giaquinta, M., Modica, G. and J. Soucek: Cartesian Currents in the Calculus of Variations. Berlin: Springer-Verlag 1998.

[15] Hang, F. and F. H. Lin: Topology of Sobolev mappings. Preprint 2000.

[16] Hardt, R. and T. Rivière: Connecting topological Hopf singularities. Preprint 2001.

[17] Morgan, F.: Geometric Measure Theory. A Beginner's Guide, 2nd ed. Boston: Acad. Press Inc. 1995. 
[18] Pakzad, M. R.: Existence of infinitely many weakly harmonic maps from a domain in $\mathbb{R}^{n}$ into $S^{2}$ for non-constant boundary data. Calc. Var. 237 (2001), 97 - 121.

[19] Pakzad, M. R.: Relaxing the Dirichlet energy for maps into $S^{2}$ in high dimensions. Comm. Cont. Math. 2000 (to appear).

[20] Pakzad, M. R. and T. Rivière: Weak density of smooth maps for the Dirichlet energy between manifolds. Preprint 2000.

[21] White, B.: Rectifiability of flat chains. Ann. Math. (2) 150 (1999), 165 - 184.

Received 01.11.2001 